\title{
Examining the impact of a social skills training program on preschoolers' social behaviors: a cluster-randomized controlled trial in child care centers
}

Marie-Pier Larose ${ }^{1}$, Isabelle Ouellet-Morin ${ }^{1}$, Francis Vergunst ${ }^{1}$, Frank Vitaro ${ }^{1}$, Alain Girard ${ }^{1}$, Richard E. Tremblay ${ }^{1}$, Mara Brendgen ${ }^{2}$ and Sylvana M. Côté $1,3,4^{*}$

\begin{abstract}
Background: Preschoolers regularly display disruptive behaviors in child care settings because they have not yet developed the social skills necessary to interact prosocially with others. Disruptive behaviors interfere with daily routines and can lead to conflict with peers and educators. We investigated the impact of a social skills training program led by childcare educators on children's social behaviors and tested whether the impact varied according to the child's sex and family socio-economic status.
\end{abstract}

Methods: Nineteen public Child Care Centers (CCC, $n=361$ children) located in low socio-economic neighborhoods of Montreal, Canada, were randomized into one of two conditions: 1$)$ intervention ( $n=10$ CCC; 185 children) or 2) wait list control ( $n=9$ CCC; 176 children). Educators rated children's behaviors (i.e., disruptive and prosocial behaviors) before and after the intervention. Hierarchical linear mixed models were used to account for the nested structure of the data.

Results: At pre-intervention, no differences in disruptive and prosocial behaviors were observed between the experimental conditions. At post-intervention, we found a significant sex by intervention interaction ( $\beta$ intervention by sex $=-1.19, p=0.04$ ) indicating that girls in the intervention condition exhibited lower levels of disruptive behaviors compared to girls in the control condition (f2 effect size $=-0.15$ ). There was no effect of the intervention for boys.

Conclusions: Girls may benefit more than boys from social skills training offered in the child care context. Studies with larger sample sizes and greater intervention intensity are needed to confirm the results.

Trial registration: Current clinical trial number is ISRCTN84339956 (Retrospectively registered in March 2017). No amendment to initial protocol.

Keywords: Childcare-based intervention, Child development, Social skills, Early childhood education, Problem behavior

\footnotetext{
* Correspondence: sylvana.cote.1@umontreal.ca

${ }^{1}$ Université de Montréal, Montreal, Canada

${ }^{3}$ Université de Bordeaux, INSERM U1219, Bordeaux, France

Full list of author information is available at the end of the article
}

\section{$\triangle B M C$}

(c) The Author(s). 2020 Open Access This article is licensed under a Creative Commons Attribution 4.0 International License, which permits use, sharing, adaptation, distribution and reproduction in any medium or format, as long as you give appropriate credit to the original author(s) and the source, provide a link to the Creative Commons licence, and indicate if changes were made. The images or other third party material in this article are included in the article's Creative Commons licence, unless indicated otherwise in a credit line to the material. If material is not included in the article's Creative Commons licence and your intended use is not permitted by statutory regulation or exceeds the permitted use, you will need to obtain permission directly from the copyright holder. To view a copy of this licence, visit http://creativecommons.org/licenses/by/4.0/ The Creative Commons Public Domain Dedication waiver (http://creativecommons.org/publicdomain/zero/1.0/) applies to the data made available in this article, unless otherwise stated in a credit line to the data. 


\section{Background}

The use of early education and care services has substantially increased over the past four decades in most Western industrialized countries [1]. Early education and care services refer to regular group-based care of children prior to school entry (i.e., under age 5 years in North America) by someone other than the parents. Group-based child care centers (CCC) are one of the most important structured environments for early child socialization. Research suggests that exposure to highquality child care in preschool settings has a positive effect on children's social and cognitive school preparedness [2-4]. Benefits are particularly evident among children raised in poverty or in a low socio-economic status (SES) families [4-7]. Attending an early education and care setting is therefore an important preventive strategy for social adjustment and academic attainment problems [3, 8].

During the preschool years, children are more likely to exhibit disruptive behaviors such as aggression, noncompliance with rules and negative affectivity especially in social settings like CCCs [9]. This is because they are required to interact with many peers and educators for many hours each day and because they have not yet acquired sufficient self-control and the social skills necessary to communicate their needs and negative emotions $[10,11]$. Emotional and cognitive immaturity in CCC settings may also be compounded by a phenomenon known as social contagion whereby preschoolers exposed to peers with disruptive behaviors mirror these behaviors or are forced to respond in similar ways in order to adapt to the social context (e.g. pushing, hitting, kicking) [12-14]. Children with disruptive behavioral problems tend to disrupt CCC daily routines, leading to conflict with peers and educators [15]. They are also more likely to be excluded from socially and cognitively stimulating activities and consequently to experience academic and social adjustment difficulties later on $[15,16]$. It is therefore vital to provide child care environments that promote the development of good social relationships with peers and educators as early as possible so that children can enter the formal education system with adequate social and cognitive abilities [17].

\section{Children at higher risk of disruptive behavior problems}

During the preschool years, boys and girls exhibit similar levels of disruptive behaviors, but males exhibit more problems after school entry [14, 18]. Studies show that early preventive interventions delivered in CCC settings can yield short- and long-term benefits [19-21]. However, the question of whether boys and girls respond differently to these interventions is not well-documented in the literature. Of five preschool intervention studies that targeted children's socio-emotional development [22], only one reported testing the interaction between the experimental conditions and the children's sex [23]. Girard and colleagues reported that an educator training intervention designed to scaffold peer interactions and use dramatic play reduced aggressive behaviors in boys but not girls [23]. This suggests that males and females may respond differently to disruptive behavioral intervention programs and further investigation of sex as a putative moderator is therefore warranted.

Another potentially important moderator of the effects of disruptive behavioral intervention programs is the SES of the child's family. Children from low-SES families are more likely to exhibit disruptive behaviors from preschool to pre-adolescence when compared with children from higher SES families [14, 24]. Consequently, children from low-SES families are more prone to enter school with socio-emotional skills deficits that undermine school adjustment [15]. However, CCC attendance may counteract the influence of a socio-economically deprived familyenvironment on children's socio-emotional skills by providing cognitive stimulation and socialization opportunities in a well-structured environment [25]. Children from low-SES families might therefore be more responsive to interventions delivered in CCC that target social-emotional skills development.

\section{Interventions on Children's social development in child care context}

Behavioral and cognitive management strategies in the context of preschools have shown positive short- and long-term effects on social behaviors, academic readiness and cognitive abilities, especially in the context of Head Start programs [20, 26-29]. However, outside of the Head Start literature, few studies have investigated the role of child care interventions on children's socioemotional development [22]. Doing so is important because the resources available to educators may vary between Head Start and community-based CCC settings. Head Start is a highly-structured government-run preschool program in which teachers have formal training in early childhood education and follow a prescribed curriculum focused on improving school readiness [30]. Community-based child care services, in contrast, may be run by public or private agencies, in which child care educators may not endorse a structured curriculum and may or may not have received formal training. Consequently, educators' capacity to effectively implement social skills programs may vary widely between these contexts.

Previous CCC interventions have typically targeted caregiver-child relationship as their active ingredient and implemented a specific curriculum, i.e., activities around a certain theme [22]. One example is the Preschool Life Skills (PLS) which focuses on thirteen skills related to instruction-following, functional communication, delay 
tolerance, and friendship. Studies show that the PLS can significantly reduce disruptive behaviors in preschool children [21]. Additionally, educators reported that the social skills training was easy to incorporate into their daily routine and improved the social dynamics between children in their groups [21]. In this project, we evaluate a social skills training similar to the PLS - the "Minipally" program - which focuses on social skills development in a group context. The Minipally program is distinct that it is oriented less towards communication skills and preparedness for the school environment, and more towards social and emotional regulation skills.

\section{Objectives}

Using a cluster-randomized controlled trial, we tested the impact of a social skills training program, delivered by child care educators, on children's disruptive and prosocial behaviors. We also examined whether children's sex and family SES moderated the impact of the program. We expected children exposed to the social skills training program to exhibit lower levels of disruptive behaviors and higher levels of prosocial behaviors at postintervention compared to children in the control condition. Given the lack of evidence showing that children's sex and family SES moderate the impact of social skills programs in CCC contexts, we did not have hypotheses about these variables.

\section{Methods}

\section{Study design}

Heads of 38 public CCC of the greater Montreal region were invited to participate in the study as they respected our eligibility criterion for participation: i.e., providing services to a minimum of $25 \%$ of children from lowincome families and being in low-SES neighborhoods. Neighborhood SES was defined according to official provincial [31] and national criteria [32]. Lower-income families were those entitled to a special government subsidy program providing free child care access for families with an annual family income below CAN\$20,000. After an information session, nineteen CCCs agreed to participate in the 8-month study. The CCCs were randomized with a 1 : 1 ratio to either: 1 ) the intervention condition (receiving the program in year 1) or 2) the wait list control condition (receiving the program in year 2) using a computergenerated randomization sequence. Each CCC included between one and 5 groups (mean $=2.32), n=8$ preschoolers led by an educator. Forty-three groups $(n=361$ children) from 19 CCCs were recruited in September 2013 and took part in the study (Fig. 1: Trial Flow Diagram). Written consent to participate in the study were obtained from parents, educators and directors of the CCCs. The study was approved by the Sainte-Justine Hospital Ethical Research Committee (ref: 2014-565, 3738) and registered on a primary clinical trial registry prior to beginning data analysis. A detailed description of the study protocol describing the rationale behind the Minipally program and its evaluation was published shortly thereafter [33].

\section{Minipally curriculum}

The Minipally program is an adaptation of an earlier social skills training programs for school-aged children - i.e. Fluppy program - which was developed by our research team and has shown long-term benefits for academic achievement, employment, income, delinquency and substance abuse [34, 35]. Over the past 20 years, experienced educational psychologists and psychoeducators have updated the Fluppy program to address the evolution of best practices in social skill training and adapt it to younger age groups, i.e. preschool-aged children. For example, in the school-aged program, children are taught how to deal with several emotions at the same time (e.g., feeling sad and upset) and to talk about their frustrations, while in the preschool version, children are taught to identify and name emotions and to manage their frustrations using age-appropriate stress-releasing techniques. Thus, while preschool-aged children are taught to use breathing techniques using the butterfly analogy, i.e. to breathe and raise their wings (arms) like a butterfly, school-aged children are taught to pause, withdraw from the situation if possible, and take five deep breaths.

The Minipally curriculum is delivered by each educator to her own group of children using a puppet via 16 play sessions over a period of 8 months. The puppet presents itself as a loyal and enthusiastic friend who visits the CCC to model prosocial behaviors and social inclusion by discussing/playing with his friends (other puppets) and with the children. The full curriculum includes generic components of social skills training programs: introduction to social contact (make and accept contact from others, make requests); problem solving (identifying the problem, generating solutions); self-regulation (deep breathing to calm down, accepting frustration, learning to share, tolerating frustration); and emotional regulation (identifying and expressing emotions, listening to the other). The skills taught in each workshop are presented in Table S1 in supplementary material.

Specifically, in each workshop, the educator calls on the Minipally puppet who then directly solicits the participation of each child and models adaptive social skills. Like children, Minipally feels great joys, but also has some difficulties with contact with others. The workshops are lively to solicit the participation and feedback of children as Minipally suggests ways for children to do things or asks them for suggestions. During the workshops, Minipally verbalizes a lot; he communicates 


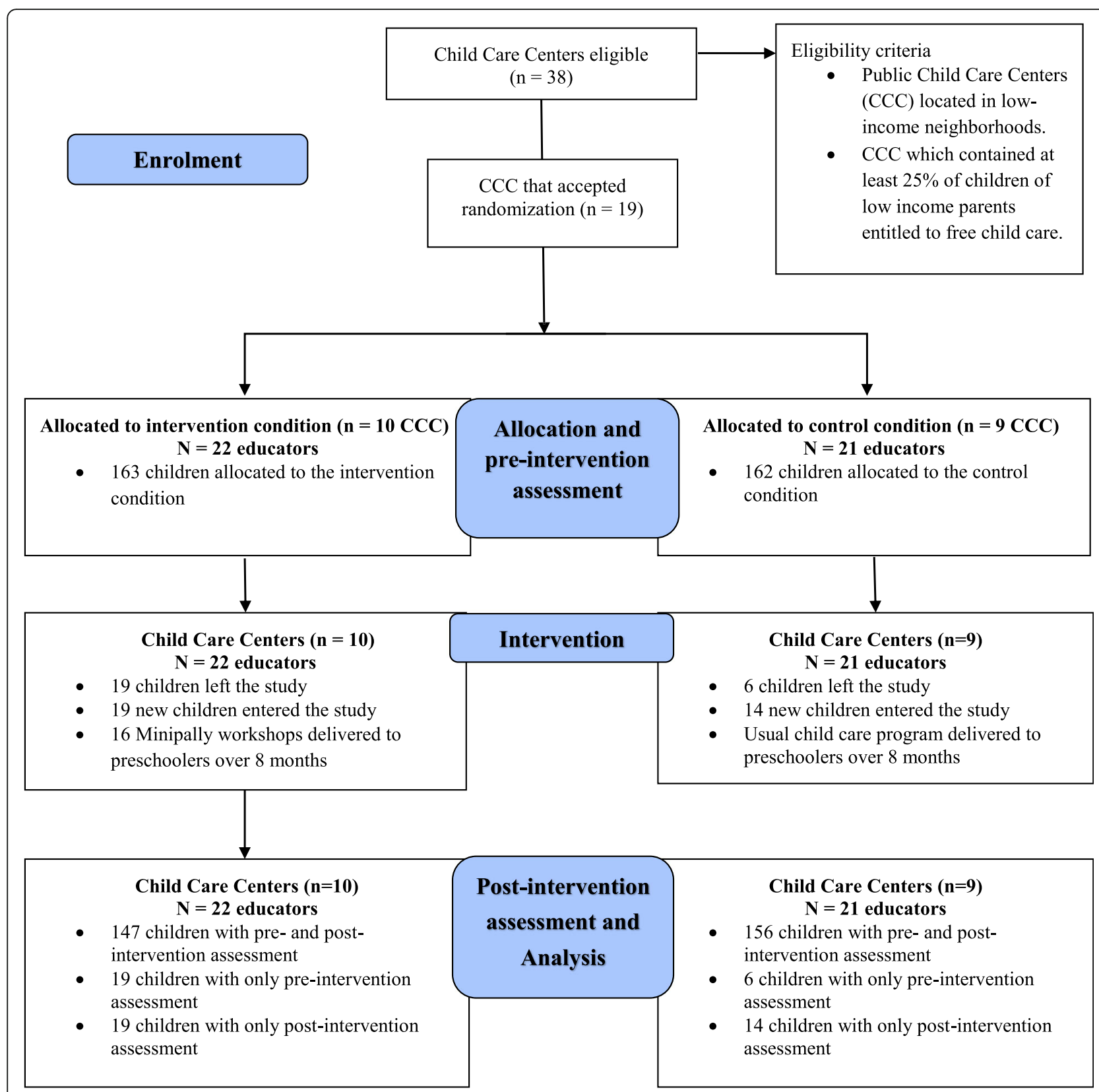

Fig. 1 Minipally Trial Flow Chart. Note. CCC = Child Care Centers

everything he thinks and does in order to help children remember his actions, words, emotions and attitudes. Minipally is very attentive throughout the workshop as he congratulates children who exhibit the desired behaviors (i.e., wait for his turn, help another child) and encourages those who make efforts to practice the new skills presented. In other words, Minipally acts as a safe and friendly figure for children and a playful tool for child care educators to introduce new concepts and rules in a group context. Child care educators are also invited to reinvest the strategies presented by Minipally in natural settings on a day to day basis: they are encouraged to observe children during free play, reinforce positive behaviors as they occur and invite children to refer to what they learned during the last Minipally visit.

\section{Educator training and supervision}

The program was implemented as follows. The 16 workshops of the Minipally curriculum were presented to the educators during a 2-day training delivered by trained professionals (i.e., psychoeducators). After the workshops the psychoeducators remained available by telephone for additional questions during the implementation of the 
curriculum by the educators. CCC directors were financially compensated for the replacement of the educators while they were trained. Next, the educators delivered the Minipally intervention over 8-months (one session every 2 weeks) and received $12 \mathrm{~h}$ (i.e., $4 \times 3$-h supervision; week 6 , 12, 18 and 24 of the trial) of group supervision. During the supervision sessions, between 8 and 10 educators met with a psychoeducator to discuss the challenges associated with the implementation of the Minipally curriculum.

\section{Measures \\ Outcomes: disruptive and prosocial behaviors assessed by educators}

Educators completed the Social Behavior Questionnaire [36] for each child in their group at pre- and postintervention. Two dimensions of the questionnaire were used: a) Disruptive Behaviors, which included five opposition items (e.g., has been defiant or has refused to comply with an adult request), four impulsivity/hyperactivity items (e.g., has had difficulty waiting for his/her turn in games) and six physical aggression items (three reactive, e.g., has reacted aggressively when teased, and three non-reactive, e.g., has gotten into fights) (Cronbach alpha = 0.86); and b) Prosocial Behaviors (e.g., has helped other children, has shared his toys with others, has comforted a child who was upset; 7 items) (Cronbach alpha = 0.79). Educators rated each item using a 3-point Likert scale according to the frequency of the behavior in the last 2 weeks $(0=$ never, $1=$ sometimes, and $2=$ often $)$. For each dimension, we created a cumulative score varying from 0 to 10 , with 0 indicating that the child did not exhibit this behavior and 10 indicating that the child often exhibits this behavior.

\section{Covariates and moderators}

\section{Family sociodemographic characteristics}

Before beginning the intervention, the child's parents completed a questionnaire about their child's CCC attendance details (e.g., number of hours per week, number of months since first attendance), the age and sex of their child, their family composition (e.g., number of siblings), and their socio-demographic background (education and income). A family SES score was then created by combining the maternal education and family income variables (i.e., total income in the household where the child lives most of the time). A low-SES score was assigned if the child lived in a household where the family earned less than CAN $\$ 20,000$ per year and where the highest level of maternal education was a high school diploma. If the child was living in a household where the family was earning more than $\mathrm{CAN} \$ 20,000$, or where the mother had obtained any training following her high school diploma, the child was assigned to the middlehigh SES group.

\section{Statistical analysis}

\section{Sample size calculation}

Prior to the recruitment, we performed an a-priori power analysis to determine the sample size needed for the trial. The mean and standard deviation estimates for preschoolers' disruptive and prosocial behaviours were taken from the Quebec Longitudinal Study of Children's Development [24]. We did not have an estimate of the intra-class correlation (ICC) for CCC, so we estimated different scenarios using $0.1,0.15$ and 0.20 as the ICC coefficient and potential effect sizes (i.e., 0.3, 0.4 and 0.5) based on the difference in mean levels of disruptive and prosocial behaviours between the intervention and control conditions. We used Heo's statistical procedure for cluster randomized trials with three-level units in our sample size estimation [37]. In other words, our calculation was based on the expected mean number of groups within each child care centers-i.e. 2 groups per child care center. Using the 0.15 ICC scenario, our power calculation indicated that 19 child care services would allow to detect a medium-size effect of the intervention on the selected outcomes, with $90 \%$ power at a 2 -sided significance level of $\alpha=5 \%$. Our model can be stated as $Y_{i j k}=$ $\beta_{0}+\delta X_{i}+u_{i}+u_{j} \quad$ (i) $+e_{i j k} ;$ where $Y_{i j k}$ is the postintervention response of the $i^{\text {th }}$ study participant in the $j^{\text {th }}$ educator group nested in the $\mathrm{k}^{\text {th }}$ child care center, $\beta_{0}$ represents the baseline value of our primary outcome, while $\delta \mathrm{X}_{\mathrm{i}}$ is the main effect of the intervention (where $\mathrm{X}=0$ for wait list group and $\mathrm{X}=1$ for the intervention group), and the last three terms are random effects at every level of the trial analysis [37]. This scenario was chosen in accordance with our financial resources and the feasibility of the study [33]. The cluster randomization ensured that children from the control wait list condition were not exposed to the intervention. After completion of data collection, all control CCC received the social skills training.

\section{Preliminary analysis \\ Randomization balance analysis}

Despite the use of a cluster randomization, there is still the possibility that individual characteristics are unequally distributed between the two experimental conditions. We therefore performed a series of preliminary analyses to compare the intervention and control conditions at baseline on a host of variables that may directly or indirectly impact the effect of the intervention (see Table 1). Only children's age, the number of months of attendance at the CCC and family SES differed between the intervention and control groups. However, these variables were not significantly associated with any of the outcomes and were therefore not included as control variables based on the randomization balance analysis. 
Table 1 Descriptive Statistics of the Sample According to Intervention Conditions

\begin{tabular}{|c|c|c|c|}
\hline & $\begin{array}{l}\text { Control } \\
(n=176)\end{array}$ & $\begin{array}{l}\text { Intervention } \\
(n=185)\end{array}$ & $p$-value \\
\hline Sex (boy) ${ }^{a}$ & $86(49.1 \%)$ & $98(52.7 \%)$ & 0.57 \\
\hline \multirow[t]{2}{*}{ Age (months) ${ }^{b}$} & $52.8(5.0)$ & $54.5(4.5)$ & $p<0.01$ \\
\hline & 164 & 165 & \\
\hline \multirow[t]{2}{*}{ Children with a developmental diagnosis ${ }^{a}$} & $7(4.3 \%)$ & $10(6.1 \%)$ & 0.47 \\
\hline & 162 & 164 & \\
\hline \multirow[t]{2}{*}{ Siblings ${ }^{a}$} & $128(78.5 \%)$ & $143(86.7 \%)$ & 0.12 \\
\hline & 163 & 165 & \\
\hline Language spoken at home ${ }^{a}$ & & & 0.56 \\
\hline French & $123(76.4 \%)$ & $117(71.3 \%)$ & \\
\hline English & $4(2.5 \%)$ & $4(2.4 \%)$ & \\
\hline \multirow[t]{2}{*}{ Other } & $34(21.1 \%)$ & $43(26.2 \%)$ & \\
\hline & 161 & 164 & \\
\hline \multirow[t]{2}{*}{ Number of months the child attended childcare ${ }^{b}$} & $39.4(9.3)$ & $40.2(9.9)$ & 0.46 \\
\hline & 151 & 159 & \\
\hline Child care hours/week ${ }^{a}$ & & & 0.26 \\
\hline Less than $30 \mathrm{~h}$ & $24(14.6 \%)$ & $35(21.2 \%)$ & \\
\hline Between 30 and $40 \mathrm{~h}$ & $104(63.4 \%)$ & 95 (57.6\%) & \\
\hline \multirow[t]{2}{*}{ More than $40 \mathrm{~h}$} & $36(22.0 \%)$ & $35(21.2 \%)$ & \\
\hline & 164 & 165 & \\
\hline Family socio-economic status ${ }^{a}$ & & & 0.04 \\
\hline Low socio-economic status & $28(18.7 \%)$ & $15(10.1 \%)$ & \\
\hline \multirow[t]{2}{*}{ Middle-high socio-economic status } & $122(81.3 \%)$ & $133(89.9 \%)$ & \\
\hline & 150 & 148 & \\
\hline
\end{tabular}

\footnotetext{
${ }^{a}$ Frequency (\%)
}

${ }^{b}$ Mean (SD)

Note1. SD = Standard deviation

Note 2. We used bivariate analyses (t-test for continuous variables and chi-square for categorical variables) to verify whether socio-demographic characteristics of the child's family were balanced between the intervention and control groups

\section{Attrition analysis}

No CCC withdrew from the study over the course of the intervention. However, 25 children left their CCC between pre- and post-intervention, representing a $7 \%$ attrition rate. These children were replaced by 33 newcomers (14 in the control condition and 19 in the intervention condition). If the new children entered their CCC in the first half of the trial (i.e., week 16 out of 32), they were included in the post-intervention assessment and in further analysis, after first obtaining parental consent. Children who entered the CCC after the 16th week of the intervention were not invited to participate in the study. In attrition analyses, we compared the 25 children who left the study with the 33 children who entered after the pre-intervention assessment (i.e., newcomers) and the 303 children who entered at pre-intervention and remained in the study. More children left the intervention condition than the control condition, but newcomers were equally distributed in both experimental conditions. There were no statistically significant differences between the children enrolled at baseline, those who left the study and those who entered later, in terms of sex, age and number of siblings. However, children who entered the intervention group later were more likely to come from middle-high SES families while children who entered the wait list group were more likely to come from low-SES families. We therefore controlled for family SES in all analyses.

\section{Are there differences between experimental conditions at pre-intervention on children's disruptive and prosocial behaviors?}

We used hierarchical linear mixed models to examine differences in disruptive and prosocial behaviors between children in the intervention and control conditions at pre-intervention. No differences were found with respect to pre-intervention disruptive and prosocial behaviors (see Supplementary material Table S2). However, girls in 
the intervention group exhibited significantly higher levels of prosocial behaviors compared to girls in the control group and compared to boys from both the intervention group and the control group, respectively $(\beta$ intervention by sex $=1.61, p<0.01)$. We therefore controlled for pre-intervention levels of children's prosocial behaviors in post-intervention models, in addition to assessing a potential moderating effect of children's sex. For disruptive behavior, we did not find any significant interaction between the experimental condition and children's sex, and consequently did not control for preintervention levels of disruptive behaviors in subsequent models.

\section{Main analysis}

Hierarchical linear mixed models were used to estimate the main effects of the intervention on post-intervention disruptive and prosocial behaviors and to estimate if the impact of the intervention varied according to children's sex and family SES. To account for variation in the number of children across CCCs, we used the restricted maximum likelihood estimator in every model. The analysis was performed in five steps.

First, because randomization was performed at the CCC level, we had to account for clustering in our data and we therefore ran an unconditional model to estimate the intra-class correlation (ICC) between clusters. The ICC is the proportion of variance in the outcome variable that is explained by the grouping structure of the hierarchical model [38]. It reports the amount of variation unexplained by any predictors in the model that can be attributed to the grouping variable, compared to the overall unexplained variance [38]. In the unconditional model, only the intercept was introduced as a fixed effect.

Second, we introduced the experimental condition variable as a main fixed predictor with and without the family SES covariate. Since the CCCs are the unit of randomization in this study, we expected variation between and within clusters and therefore accounted for this by introducing random effects. In other words, because children's sex and family SES could vary within the same cluster, i.e., children from different SES backgrounds attended the same CCC, we introduced them as fixed and random effects for the adjusted and interaction models.

In subsequent models, we added an interaction term between our hypothesized moderators (i.e., children's sex and family SES) and the experimental condition variable in the prediction of children's disruptive and prosocial behaviors. Once again, the random effects specified in these models were the intercept, as well as family SES and children's sex. Because of baseline differences between the experimental conditions found in preliminary analysis, we also added children's preintervention prosocial behavior score as a fixed and random effect when assessing the moderating effect of children's sex on the association between the experimental condition and post-intervention prosocial behavior.

Fourth, we performed pairwise comparisons between the intervention and the control group according to children's sex and family SES, based on the mixed hierarchical model mean estimates. Finally, we estimated the effect sizes of the difference in means using the $f 2$ fixed effect size estimation [39] for hierarchical linear mixed models recommended by Lorah (2018) [40]. The $f 2$ effect size statistic represents the proportion of variance explained by the given fixed effects relative to the unexplained proportion of outcome variance. Effects of 0.02 , 0.15 and 0.35 are considered small, medium and large respectively [41].

\section{Results \\ Descriptive statistics \\ Participants}

Children $(n=361)$ were distributed into 19 different CCCs. Table 1 shows that most children attended CCC for 30 to $40 \mathrm{~h}$ per week and that the number of boys and girls in the intervention group and the control group was roughly equal. Table 2 shows children's raw scores for disruptive and prosocial behaviors at pre- and postintervention according to the experimental conditions.

\section{Implementation of Minipally}

All educators were female, and most (85\%) had a professional early education training. All educators in the intervention group received the two-day Minipally training. Implementation was monitored throughout the year via four half-day supervision sessions. At the last supervision session (week 24 out of 32 in the trial), all educators in the intervention group had implemented 12 of the 16 Minipally workshops. Thereafter, the exact number of workshops conducted by every educator was not monitored.

\section{Did the intervention have an impact on children's social skills? \\ Disruptive behaviors}

At post-intervention, the unconditional model showed that about $9 \%$ of the total variation in post-intervention disruptive behaviors was accounted for by differences between CCCs. When entering the experimental condition variable as a fixed effect, while adjusting for children's family SES $(\beta=0.27, p=0.52)$, we found no main effect of the intervention on children's post-intervention disruptive behaviors $(\beta=0.39, p=0.34)$. This suggested that the mean level of post-intervention disruptive behaviors was not different between the intervention and 
Table 2 Levels of Disruptive and Prosocial Behaviors by Intervention Conditions and Time of Assessment

\begin{tabular}{llllll}
\hline & \multicolumn{2}{l}{ Control } & & \multicolumn{2}{l}{ Intervention } \\
\cline { 2 - 3 } Dependent variables & Pre-intervention & Post-intervention & & Pre-intervention & Post-intervention \\
\hline Disruptive behaviors $^{{ }^{2}}$ & $3.43(0.20)$ & $3.47(0.28)$ & $3.07(0.20)$ & $2.94(0.27)$ \\
Prosocial behaviors $^{{ }^{2}}$ & $6.46(0.24)$ & $6.84(0.22)$ & $6.45(0.24)$ & $7.31(0.22)$ \\
\hline
\end{tabular}

a Mean (SD)

Note. $\mathrm{SD}=$ Standard deviation. Pre-intervention assessment was conducted in October and post-intervention assessment in June the following year

the control group. The ICC for this model dropped to 0.05 , indicating that we accounted for a larger portion of the variation among the different CCCs and that less variation existed in the random intercepts of our model. Coefficients for the post-intervention models and their associated ICCs are presented in Table 3.

\section{Did child's sex or the socio-economic status of the family moderate the impact of the intervention?}

We found a significant interaction between experimental conditions and children's sex $(\beta=-1.19, p=0.04$, Fig. 2a), indicating lower levels of post-intervention disruptive behaviors in the intervention group compared to the control group for girls $(F=4.19, d f=43.08, p=0.04$; $f 2$ effect size $=-0.15)$. For boys, there was no difference in post-intervention disruptive behaviors between the intervention group and the control group $(F=0.37, d f=$ 49.20, $p=0.55$; $f 2$ effect size $=0.04$ ).

We also investigated the potential moderating effect of family SES, but no significant interaction was found $(\beta=$ $0.17, p=0.86$; f2 effect size for middle-high SES children $<0.01$, f2 effect size low SES $<0.01$ ).

\section{Prosocial behaviors}

For prosocial behaviors, there was no main effect of the intervention and no moderation effect of children's sex or family SES. Coefficients and ICCs for all tested models are presented in Table 3 . Figure $2 \mathrm{~b}$ shows the prosocial scores according to experimental conditions and children's sex.

\section{Sensitivity analysis}

We performed the same set of analyses with a restricted sample of children who had both pre- and postintervention assessments (i.e., newcomers were excluded from the sensitivity analysis). We found the same patterns of results, namely that the intervention led to a decrease in disruptive behaviors among girls only but had no impact on prosocial behaviors for girls or boys.

\section{Discussion}

This study used a cluster-randomized controlled trial design to test the impact of a social skills training program on children's social behaviors in Child Care Centers in low-SES neighborhoods. Using hierarchical linear mixed models, we found that the sex of the child moderated the impact of the social skills training program, reducing the level of disruptive behaviors for girls but not for boys. The failure to find an effect for prosocial behaviors may be due to the high levels of prosocial behaviors in the experimental conditions at pre-intervention, leaving little room for improvement (i.e., ceiling effects). Furthermore, we found no evidence that the SES of the child's family moderated the impact of the intervention.

\section{Examination of the evaluated intervention}

With respect to disruptive behaviors, our results are consistent with earlier findings from a similar social skills intervention developed by our research team for school-aged children- the "Fluppy program" [42] which found that disruptive behaviors at the end of the 8-month intervention were reduced for girls but not for boys [42]. One explanation for the observed sex differences is the highly verbal nature of these interventions. Sex differences in children's verbal abilities are welldocumented, particularly early in development [43, 44], so it is possible that the content and delivery of the interventions were not sufficiently accessible to boys. Indeed, the Minipally and Fluppy programs are specifically designed to improve social skills that frequently depend on verbal skills such as the ability to articulate questions or describe emotions.

Thus, while girls might be receptive to educator-led workshops that focus on enhancing social skills and reducing disruptive behaviors, this might not be the best approach for boys, who might instead benefit from educator-led dramatic play sessions, stronger educatorchild relationships, and supervised peer play to scaffold social competences [23, 45, 46]. More broadly, our results corroborate the hypothesis that children's sex is an important moderator of the impact of a social skills training program during early childhood and possibly later.

A further consideration for future studies is that adding a parenting component to the Minipally program could increase its impact. According to a recent meta-analysis, interventions with a parent component, either alone or in combination with other components, are more likely to benefit children who exhibit high levels of behavioral problems [47]. Future studies should therefore examine the unique and combined impact of child care-based and parenting-based interventions on children's social behaviors 
Table 3 Linear Mixed Models Linking Intervention Conditions to Disruptive and Prosocial Behaviors in Post-intervention

\begin{tabular}{|c|c|c|c|c|c|c|c|c|c|c|}
\hline & \multicolumn{5}{|c|}{ Intervention } & \multicolumn{5}{|c|}{ Intervention \& Covariates } \\
\hline & $\bar{\beta}$ & SE & Df & $p$-value & $\overline{I C C}$ & $\bar{\beta}$ & SE & Df & $p$-value & ICC \\
\hline \multicolumn{11}{|l|}{ Disruptive Behavior } \\
\hline \multicolumn{11}{|l|}{ Unconditional model } \\
\hline \multicolumn{11}{|l|}{ Random intercept } \\
\hline Intercept & 3.19 & 0.2 & 17.55 & $<0.01$ & 0.09 & & & & & \\
\hline \multicolumn{11}{|l|}{ Conditional Models } \\
\hline \multicolumn{11}{|l|}{ Covariate \& intervention variables } \\
\hline Intercept & 2.94 & 0.27 & 17.43 & $<0.01$ & 0.08 & 2.99 & 0.29 & 18.52 & $<0.01$ & 0.05 \\
\hline Intervention & 0.52 & 0.39 & 16.31 & 0.19 & & 0.39 & 0.40 & 18.27 & 0.34 & \\
\hline Familial SES & & & & & & 0.27 & 0.42 & 98.93 & 0.52 & \\
\hline \multicolumn{11}{|l|}{ Moderation models } \\
\hline \multicolumn{11}{|l|}{ Children's sex } \\
\hline Intercept & & & & & & 2.52 & 0.34 & 39.80 & $<0.01$ & 0.03 \\
\hline Intervention & & & & & & 0.98 & 0.46 & 36.72 & 0.04 & \\
\hline Familial SES & & & & & & 0.29 & 0.40 & 156.87 & 0.02 & \\
\hline Children's sex & & & & & & 0.93 & 0.41 & 118.14 & 0.46 & \\
\hline Children's sex * Intervention & & & & & & -1.19 & 0.57 & 113.14 & 0.04 & \\
\hline \multicolumn{11}{|l|}{ Familial SES } \\
\hline Intercept & & & & & & 3.01 & 0.30 & 17.86 & $<0.01$ & 0.05 \\
\hline Intervention & & & & & & 0.35 & 0.43 & 17.47 & 0.41 & \\
\hline Familial SES & & & & & & 0.17 & 0.67 & 146.49 & 0.80 & \\
\hline Familial SES * Intervention & & & & & & 0.17 & 0.86 & 110.96 & 0.84 & \\
\hline \multicolumn{11}{|l|}{ Prososcial Behavior } \\
\hline \multicolumn{11}{|l|}{ Unconditional model } \\
\hline \multicolumn{11}{|l|}{ Random intercept } \\
\hline Intercept & 7.08 & 0.16 & 14.95 & $<0.01$ & 0.08 & & & & & \\
\hline \multicolumn{11}{|l|}{ Conditional Models } \\
\hline \multicolumn{11}{|l|}{ Covariate \& intervention variables } \\
\hline Intercept & 7.31 & 0.22 & 14.52 & $<0.01$ & 0.07 & 7.28 & 0.23 & 17.76 & $<0.01$ & 0.03 \\
\hline Intervention & -0.47 & 0.31 & 13.44 & 0.16 & & -0.46 & 0.31 & 17.45 & 0.16 & \\
\hline Familial SES & & & & & & -0.04 & 0.35 & 109.28 & 0.92 & \\
\hline \multicolumn{11}{|l|}{ Moderation models } \\
\hline \multicolumn{11}{|l|}{ Children's sex } \\
\hline Intercept & & & & & & 4.22 & 0.46 & 167.29 & $<0.01$ & $<0.01$ \\
\hline Children's pre-intervention level of prosocial behaviours & & & & & & 0.46 & 0.06 & 144.98 & $<0.01$ & \\
\hline Intervention & & & & & & -0.17 & 0.37 & 251.65 & 0.65 & \\
\hline Familial SES & & & & & & 0.09 & 0.3 & 20.02 & 0.77 & \\
\hline Children's sex & & & & & & 0.31 & 0.32 & 251.81 & 0.34 & \\
\hline Children's sex * Intervention & & & & & & -0.24 & 0.42 & 251.25 & 0.57 & \\
\hline \multicolumn{11}{|l|}{ Familial SES } \\
\hline Intercept & & & & & & 7.34 & 0.23 & 18.19 & $<0.01$ & 0.03 \\
\hline Intervention & & & & & & -0.48 & 0.34 & 17.72 & 0.17 & \\
\hline Familial SES & & & & & & -0.06 & 0.45 & 110.91 & 0.89 & \\
\hline Familial SES * Intervention & & & & & & -0.07 & 0.61 & 94.10 & 0.91 & \\
\hline
\end{tabular}

Note. SES Socio-Economic Status, B Regression Coefficient, SE Standard Error, Df Degree of Freedom, ICC Intra-Class Correlation 

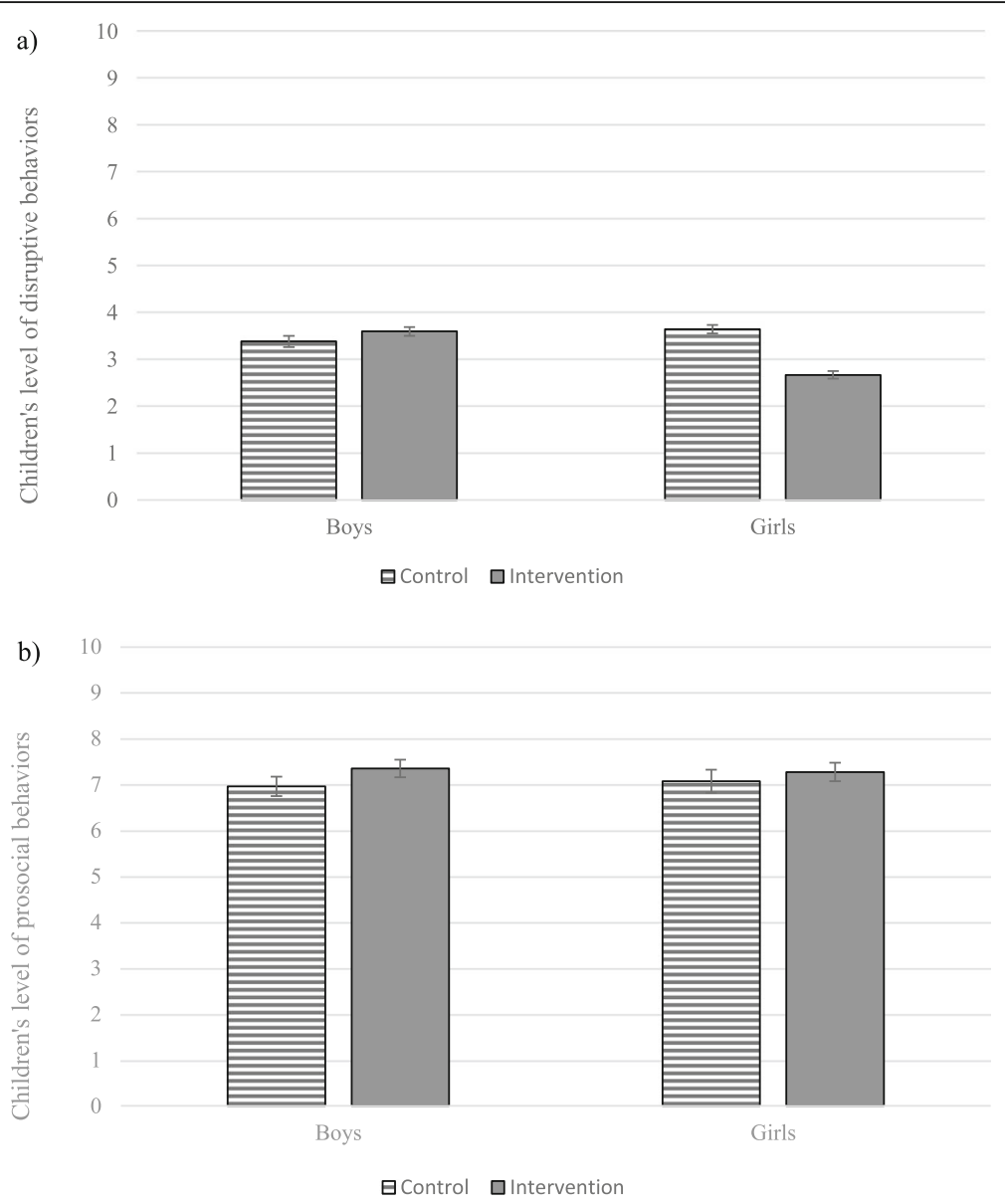

Fig. 2 Children's Levels of Disruptive (a) and Prosocial (b) Behavior in Post-intervention. Note 1. Mean score and 95\% confidence intervals on children's levels of disruptive (a) and prosocial (b) behavior in post-intervention according to intervention conditions and children's sex. Note 2. Models adjusted for children's family socio-economic status

when designing new interventions and early childhood politics.

Finally, previous work shows that social skills training programs for childhood disruptive behaviors are effective only if they are of moderate-to-high intensity [47]. It is possible that our intervention lacked the intensity necessary to significantly increase children's prosocial behaviors and reduce disruptive behaviors in boys. The educators in our trial conducted at least 12 out of 16 workshops in the Minipally child curriculum, but their reinvestment activities (i.e., follow-up activities throughout the week) were not monitored. A higher intensity intervention with systematic reinvestment activities would arguably have had a greater impact on children's social skills, especially for those exposed to risk factors in their home environment.

\section{Strengths and limitations}

The strengths of this study are its cluster-randomized experimental design, low level of cluster $(0 \%)$ and individual attrition (7\%), and the use of hierarchical linear mixed models, which accounted for the nested structure of randomization. The study had good ecological validity. It was implemented in community-based CCCs by educators who, apart from receiving a 2-day training and $12 \mathrm{~h}$ of supervision for the social skills program, had only a two-year professional degree (after high school) in early childhood and child care education.

The study has several limitations. First, we underestimated the ICC of the data in our sample size calculation, which, when combined with our modest sample size, limited our capacity to detect small effects. Future studies should replicate the intervention using larger samples and test a putative interaction with children's sex and family SES, as well as other potential moderators, such as children's baseline levels of prosocial and disruptive behaviors. Second, children's behavioral questionnaires were completed by the educator who also delivered the Minipally program. Childcare educators are a reliable source of information on disruptive behaviors because of their established ability to distinguish between normative and atypical behaviors[48, 49]; However, since the educators were involved in both the implementation of the 
intervention and the pre- and post-intervention behavioral assessments, this may have introduced a bias. For instance, due to their proximity to the project, educators in the intervention group may have noticed smaller improvement in children's behaviors than educators in the control group. Nevertheless, it is unlikely that such bias would explain the different impact of the intervention on disruptive behaviors between boys and girls. The decision to rely on the CCC educators who participated in the study was based on extensive literature that shows there is only weak to moderate agreement in social skills evaluations between raters [50]. Social skills are highly context specific, and the skills necessary to function at home are considerably different from those required in group contexts typical of CCC settings [50]. Future studies seeking to replicate our intervention should consider evaluating children's social competences based on assessments performed by independent raters. The use of objective tests - for example "The white crayon does not work ..." task by Ostrov et al. [51] in which children are asked to participate in a group drawing exercise should be considered in future studies to examine the impact of a social skills training program on children's social behaviors. Also, a follow-up assessment at school entry with kindergarten teachers who have not been involved in the project may yield more reliable results. Finally, we did not track the number of workshops implemented by child care educators - we only know that all educators performed 12 or more of the 16 workshops during the implementation year. Future studies should include a comprehensive implementation and content validity evaluation.

\section{Conclusions}

CCCs provide one of the earliest opportunities to equip children with social skills that will benefit them for the rest of their lives [52]. This study adds to a small but growing body of literature suggesting there may be important sex differences in children's responsiveness to early psychosocial interventions. Preschool programs that provide social skills training with higher intensity, a defined educative curriculum, and parent engagement may help reduce behavior problems and enhance social skills with long-term benefits to individuals and society.

\section{Supplementary information}

Supplementary information accompanies this paper at https://doi.org/10. 1186/s40359-020-00408-2.

\section{Additional file 1: Table S1. Skills taught in the Minipally program by} workshops.

Additional file 2: Table S2. Linear Mixed Models Linking Intervention Conditions to Disruptive and Prosocial Behaviors in Pre-intervention.

\section{Abbreviations}

CCC: Child care centers; ICC: Intra-class correlation; PLS: Preschool life skills; SES: Socio-economic status

\section{Acknowledgements}

We are grateful to the participants who have given their time to take part in this study. IOM holds a Canada Research Chair in the Developmental Origins of Vulnerability and Resilience. MPL is supported by a Fonds de Recherche Québécois en Santé (FRQS) doctoral fellowship. FV is supported by a FRQS post-doctoral fellowship.

\section{Authors' contributions}

The Research Unit on Children's Psychosocial Maladjustment (GRIP) provided data collection and management. SMC, MPL, IOM, FV, RET, and MB conceived and designed the study. MPL, IOM and AG analyzed and interpreted the data. SMC, MPL, FV and IOM drafted the manuscript. FV, FV RET, $A G$, and $M B$ reviewed the manuscript and had a major contribution in editing the manuscript. All authors read and approved the final manuscript after revising it critically for important intellectual content. All authors agreed to be accountable for all aspects of the work.

\section{Funding}

This study was supported by grants from the Quebec Research Fund for Society and Culture (2015-RG-178735), Canadian Institutes of Health Research (MOP: 114984), and CHU Sainte-Justine philanthropic donation (\#6483). The funding agencies had no role in the design of the study, its execution, analyses, and the interpretation of data.

\section{Availability of data and materials}

The datasets generated and/or analyzed during the current study are not expected to be available in accordance with the ethical approval received from the Ethical Research Committee: CHU Saint-Justine for confidentiality reasons.

\section{Ethics approval and consent to participate}

All procedures performed in studies involving human participants were in accordance with the ethical standards of the institutional and/or national research committee and with the 1964 Helsinki declaration and its later amendments or comparable ethical standards. The Sainte-Justine Hospital Ethical Research Committee approved all procedures in May 2013 ref.: 2014565,3738 and renewed the ethic approval every year since then. Written consent to participate in the study were obtained from parents, educators and directors of the child care centers.

Consent for publication

Written consent to publish the results in was obtained from parents, educators and head of the CCS.

\section{Competing interests}

The authors declare that they have no competing interest.

\section{Author details}

${ }^{1}$ Université de Montréal, Montreal, Canada. ${ }^{2}$ Université du Québec à Montréal, Montreal, Canada. ${ }^{3}$ Université de Bordeaux, INSERM U1219, Bordeaux, France. ${ }^{4}$ Centre de recherche du CHU Ste-Justine, Montréal, Canada.

Received: 16 July 2019 Accepted: 13 April 2020

Published online: 23 April 2020

References

1. OECD. PF3.2: enrolment in childcare and pre-scools. Paris: OECD Publishing 2014

2. Huang FL, Invernizzi MA, Drake EA. The differential effects of preschool: evidence from Virginia. Early Child Res Q. 2012;27(1):33-45.

3. Moore JE, Cooper BR, Domitrovich CE, Morgan NR, Cleveland MJ, Shah H, et al. The effects of exposure to an enhanced preschool program on the social-emotional functioning of at-risk children. Early Child Res Q. 2015;32: 127-38.

4. Laurin JC, Geoffroy MC, Boivin M, Japel C, Raynault MF, Tremblay RE, et al. Child care services, socioeconomic inequalities, and academic performance. Pediatrics. 2015;136(6):1112-24.

5. Anthony BJ, Anthony LG, Morrel TM, Acosta M. Evidence for social and behavior problems in low-income, urban preschoolers: effects of site, classroom, and teacher. J Youth Adolesc. 2005;34(1):31-9. 
6. Geoffroy MC, Cote SM, Giguere CE, Dionne G, Zelazo PD, Tremblay RE, et al. Closing the gap in academic readiness and achievement: the role of early childcare. J Child Psychol Psychiatry. 2010;51(12):1359-67.

7. Borge Al, Rutter M, Côté S, Tremblay RE. Early childcare and physical aggression: differentiating social selection and social causation. J Child Psychol Psychiatry. 2004:45(2):367-76.

8. Barnett WS. Effectiveness of early educational intervention. Science. 2011; 333(6045):975-8.

9. Boivin M, Vitaro F, Poulin F. In: Press TG, editor. Peer relationships and the development of aggressive behavior in early childhood. New York: The Gilford Press; 2005. p. 376-97.

10. Groeneveld MG, Vermeer H, van lizendoorn MH, Linting M. Children's wellbeing and cortisol levels in home-based and center-based childcare. Early Child Res Q. 2010;25(4):502-14.

11. Vermeer $\mathrm{HJ}$, van lizendoorn MH. Children's elevated cortisol levels at daycare: a review and meta-analysis. Early Child Res Q. 2006;21 (3):390-401.

12. Ribeiro LA, Zachrisson HD. Peer effects on aggressive behavior in norwegian child care centers. Child Dev. 2019;90(3):876-93.

13. Snyder J, Schrepferman L, Oeser J, Patterson G, Stoolmiller M, Johnson K, et al. Deviancy training and association with deviant peers in young children: occurrence and contribution to early-onset conduct problems. Dev Psychopathol. 2005;17(2):397-413.

14. Tremblay RE, Vitaro F, Côté SM. Developmental origins of chronic physical aggression: a bio-psycho-social model for the next generation of preventive interventions. Annu Rev Psychol. 2018:69:383-407.

15. Fantuzzo J, Bulotsky-Shearer R, McDermott PA, McWayne C, Frye D, Perlman S. Investigation of dimensions of social-emotional classroom behavior and school readiness for low-income urban preschool children. Sch Psychol Rev. 2007;36(1):44-62.

16. Sroufe LA. Emotional development: the organization of emotional life in the early years. Cambridge: Cambridge University Press; 1997.

17. Diamond A. Executive functions. Annu Rev Psychol. 2013;64:135-68.

18. Bartels M, Hendriks A, Mauri M, Krapohl E, Whipp A, Bolhuis K, et al. Childhood aggression and the cooccurrence of behavioural and emotional problems: results across ages 3-16 years from multiple raters in six cohorts in the EUACTION project. Europeanchild \& adolescent psychiatry. 2018;27(9):1105-21.

19. Lee R, Zhai F, Brooks-Gunn J, Han W-J, Waldfogel J. Head start participation and school readiness: evidence from the early childhood longitudinal study-birth cohort. Dev Psychol. 2014;50(1):202.

20. Zhai F, Raver CC, Jones SM. Social and emotional learning services and child outcomes in third grade: evidence from a cohort of head start participants. Child Youth Serv Rev. 2015;56:42-51.

21. Hanley GP, Heal NA, Tiger JH, Ingvarsson ET. Evaluation of a classwide teaching program for developing preschool life skills. J Appl Behav Anal. 2007:40(2):277-300.

22. Werner $C D$, Linting $M$, Vermeer HJ, Van IJzendoorn MH. Do intervention programs in child care promote the quality of caregiver-child interactions? A meta-analysis of randomized controlled trials. Prev Sci. 2016;17(2):259-73.

23. Girard L-C, Girolametto L, Weitzman E, Greenberg J. Training early childhood educators to promote peer interactions: effects on children's aggressive and prosocial behaviors. Early Educ Dev. 2011;22(2):305-23.

24. Côté SM, Vaillancourt T, LeBlanc JC, Nagin D, Tremblay RE. The development of physical aggression from toddlerhood to pre-adolescence: a nation wide longitudinal study of Canadian children. J Abnorm Child Psychol. 2006;34(1):68-82.

25. Felfe C, Lalive R. Does early child care affect children's development? Public Econ. 2018;159:33-53.

26. Rusby JC, Jones LB, Crowley R, Smolkowski K. An efficacy trial of Carescapes: home-based child-care practices and children's social outcomes. Child Dev. 2016;87(4):1291-310

27. Bierman KL, Domitrovich CE, Nix RL, Gest SD, Welsh JA, Greenberg MT, et al. Promoting academic and social-emotional school readiness: the head start REDI program. Child Dev. 2008;79(6):1802-17.

28. Raver CC, Jones SM, Li-Grining C, Zhai F, Bub K, Pressler E. CSRP's impact on low-income preschoolers' preacademic skills: self-regulation as a mediating mechanism. Child Dev. 2011;82(1):362-78.

29. Reinke WM, Herman KC, Dong N. The incredible years teacher classroom management program: outcomes from a group randomized trial. Prev Sci. 2018:1-12.

30. Gibbs C, Ludwig J, Miller DL. Does head start do any lasting good? Cambridge: National Bureau of Economic Research; 2011.
31. Ministère du Revenu du Québec. Child assistance program. City of Quebec: Government of the province of Quebec; 2013 .

32. Canada S. Census and 2011 National Household Survey. Canada: Statistic Canada; 2011.

33. Côté LM-P, Geoffroy MC, Laurin J, Vitaro F, Tremblay RE, et al. Testing the impact of a social skill training versus waiting list control group for the reduction of disruptive behaviors and stress among preschool children in child care: the study protocol for a cluster randomized trial. BMC psychology. 2017;5(1):29.

34. Castellanos-Ryan N, Séguin JR, Vitaro F, Parent S, Tremblay RE. Impact of a 2-year multimodal intervention for disruptive 6-year-olds on substance use in adolescence: randomised controlled trial. Br J Psychiatry. 2013;203(3):18895.

35. Tremblay RE, Kurtz L, Mâsse LC, Vitaro F, Pihl RO. A bimodal preventive intervention for disruptive kindergarten boys: its impact through midadolescence. J Consult Clin Psychol. 1995;63:560-8.

36. Tremblay RE, Vitaro F, Gagnon C, Piché C, Royer N. A prosocial scale for the preschool behaviour questionnaire: concurrent and predictive correlates. Int J Behav Dev. 1992;15(2):227-45.

37. Heo M, Leon AC. Statistical power and sample size requirements for three level hierarchical cluster randomized trials. Biometrics. 2008;64(4):1256-62.

38. Hox J, Moerbeek M, Van de Schoot R. Multilevel Analysis: Techniques and Applications, Third Edition. New York: Routledge; 2018.

39. Aiken LS, West SG, \& Reno RR. Multiple Regression: Testing and Interpreting Interactions. New York: Sage; 1991.

40. Lorah J. Effect size measures for multilevel models: definition, interpretation, and TIMSS example. Large-scale Assessments Educ. 2018;6(1):8.

41. Cohen J. A power primer. Psychol Bull. 1992;112(1):155.

42. Poulin F, Capuano F, Vitaro F, Verlaan P, Brodeur M, Giroux J. Large-scale dissemination of an evidence-based prevention program for at-risk kindergarteners. In: Promoting school readiness and early learning; 2014. p. 322-4.

43. Lange BP, Euler HA, Zaretsky E. Sex differences in language competence of 3-to 6-year-old children. Appl Psycholinguist. 2016;37(6):1417-38.

44. Toivainen T, Papageorgiou KA, Tosto MG, Kovas Y. Sex differences in nonverbal and verbal abilities in childhood and adolescence. Intelligence. 2017; 64:81-8.

45. Boisjoli R, Vitaro F, Lacourse E, Barker ED, Tremblay RE. Impact and clinical significance of a preventive intervention for disruptive boys: 15-year followup. Br J Psychiatry. 2007;191(5):415-9.

46. Vancraeyveldt $C$, Verschueren $K$, Wouters $S$, Van Craeyevelt $S$, Van den Noortgate W, Colpin H. Improving teacher-child relationship quality and teacher-rated behavioral adjustment amongst externalizing preschoolers: effects of a two-component intervention. J Abnorm Child Psychol. 2015; 43(2):243-57.

47. Epstein RA, Fonnesbeck C, Potter S, Rizzone KH, McPheeters M. Psychosocial interventions for child disruptive behaviors: a meta-analysis. Pediatrics. 2015; 136(5):947-60.

48. Verhulst FC, Koot HM, Van der Ende J. Differential predictive value of parents' and teachers' reports of children's problem behaviors: a longitudinal study. J Abnorm Child Psychol. 1994;22(5):531-46.

49. Pingault J-B, Tremblay RE, Vitaro F, Carbonneau R, Genolini C, Falissard B, et al. Childhood trajectories of inattention and hyperactivity and prediction of educational attainment in early adulthood: a 16-year longitudinal population-based study. Am J Psychiatry. 2011;168(11):1164-70.

50. Gresham FM, Elliott SN, Cook CR, Vance MJ, Kettler R. Cross-informant agreement for ratings for social skill and problem behavior ratings: an investigation of the social skills improvement system—rating scales. Psychol Assess. 2010;22(1):157.

51. Ostrov JM, Woods KE, Jansen EA, Casas JF, Crick NR. An observational study of delivered and received aggression, gender, and social-psychological adjustment in preschool:"this white crayon doesn't work ... ". Early Child Res O. 2004:19(2):355-71.

52. Denham SA, Bassett HH, Zinsser K, Wyatt TM. How preschoolers' socialemotional learning predicts their early school success: developing theorypromoting, competency-based assessments. Infant Child Dev. 2014;23(4): $426-54$

\section{Publisher's Note}

Springer Nature remains neutral with regard to jurisdictional claims in published maps and institutional affiliations. 